\title{
CORRECTIONS
}

\section{Journal withdraws article after complaints from drug manufacturers}

This News story by Nigel Hawkes (BMJ 2011;342:d2335, doi:10.1136/bmj.d2335) wrongly states that Novo Nordisk manufactures the drug exenatide (marketed as Byetta). In fact, the drug is manufactured by Amylin Pharmaceuticals and Eli Lilly.

Cite this as: $B M J 2011 ; 342: \mathrm{d} 2513$ 